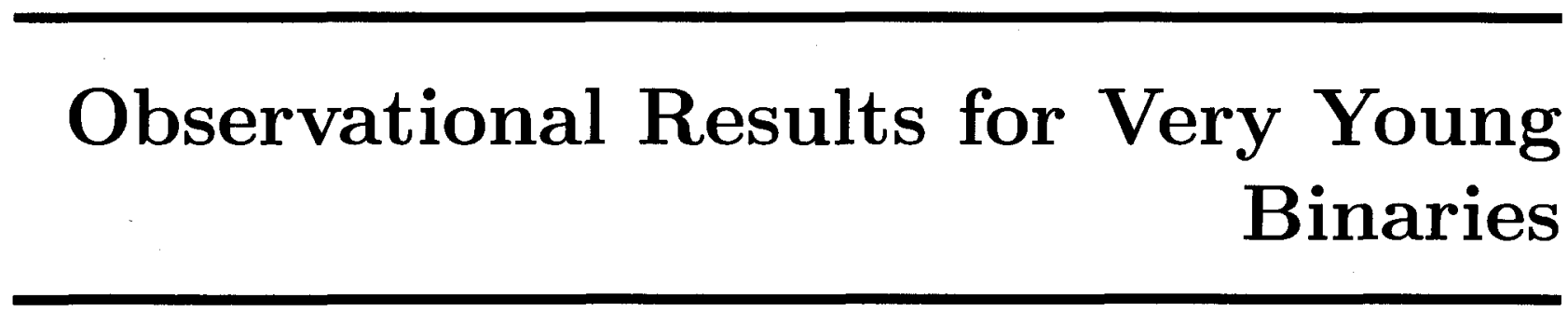




\title{
ORBITAL MOTIONS IN BINARY PROTOSTELLAR SYSTEMS
}

\author{
L. F. Rodríguez ${ }^{1}$ \\ RESUMEN
}

Mediante observaciones de alta resolución con el VLA en múltiples épocas hemos detectado movimientos orbitales en varios sistemas protobinarios de baja luminosidad en los complejos moleculares de Taurus y $\rho$ Ophiuchus. Las masas que se obtienen con la tercera ley de Kepler son del orden de 0.5 a $2 M_{\odot}$, como se espera para protoestrellas de baja masa. Las luminosidades bolométricas -relativamente altas- de estos sistemas jóvenes corroboran el concepto de que las protoestrellas derivan su luminosidad principalmente de la acreción, y no de las reacciones nucleares. Además, en una de las fuentes estudiadas (un sistema múltiple en Taurus) una estrella jóven de baja masa ha mostrado un cambio drástico en su órbita, después de un encuentro cercano con otra componente del sistema, que supuestamente es una binaria. El gran movimiento propio adquirido por esta protoestrella de baja masa $\left(20 \mathrm{~km} \mathrm{~s}^{-1}\right)$ sugiere una expulsión del sistema.

\section{ABSTRACT}

Using high-resolution $\left(\sim 0{ }^{\prime \prime} 1\right)$, multi-epoch Very Large Array observations, we have detected orbital motions in several low-luminosity protobinary systems in the Taurus and $\rho$ Ophiuchus molecular complexes. The masses obtained from Kepler's third law are of the order of 0.5 to $2 M_{\odot}$, as expected for such low-mass protostars. The relatively large bolometric luminosities of these young systems corroborates the notion that protostars obtain most of their luminosity from accretion and not from nuclear reactions. In addition, in one of the sources studied (a multiple system in Taurus), a low-mass young star has shown a drastic change in its orbit after a close approach with another component of the system, presumed to be a double star. The large proper motion achieved by this low mass protostar $\left(20 \mathrm{~km} \mathrm{~s}^{-1}\right)$, suggests an ejection from the system.

Key Words: ASTROMETRY - BINARIES: GENERAL — STARS: PRE-MAIN SEQUENCE

\section{INTRODUCTION}

It is well known that the study of orbital motions in binary systems is perhaps the most reliable way of determining the masses of main sequence stars. This fundamental work has been going on in the optical band for about 200 years. Recently, speckle and adaptive optics observations in the near-IR have been used successfully to investigate the proper motions and masses of $\mathrm{T}$ Tauri binary systems (e. g. Ghez et al. 1995).

What about young stellar systems that are so obscured that they cannot be detected even at nearIR wavelengths? In the last few years our group and other researchers have used radio observations of a handful of binary systems that are generally detected only at these long wavelengths. Here we present a review of these results.

\section{DETECTING STARS AT RADIO WAVELENGTHS}

Remarkably, even when a normal stellar photosphere located at interstellar distances is not detectable at radio wavelengths, there are at least three

\footnotetext{
${ }^{1}$ Centro de Radiostronomía y Astrofísica, UNAM, Morelia, México.
}

mechanisms that allow tracking the position of a star in the radio:

- Gyrosynchrotron emission from an active magnetosphere.

- Free-free emission from an ionized wind or jet.

- Thermal emission from dust in a circumstellar disk.

Of these three mechanisms, the first one is the best for astrometric purposes since the emission originates from a region comparable in size to the star. The last two mechanisms originate from regions that extend in the order of a few to a few tens of $\mathrm{AU}$ and asymmetries in the regions of emission can mimic the effect of apparent motions in the analysis.

\section{OBSERVED SOURCES}

In this section we will summarize the results obtained up to now. We will first discuss three sources that apparently are binaries: L1551 IRS5, YLW 15, and L1527.

\subsection{L1551 IRS5}

L1551 IRS5 is one of the prototypical young stellar sources. In the centimeter radio continuum it has 

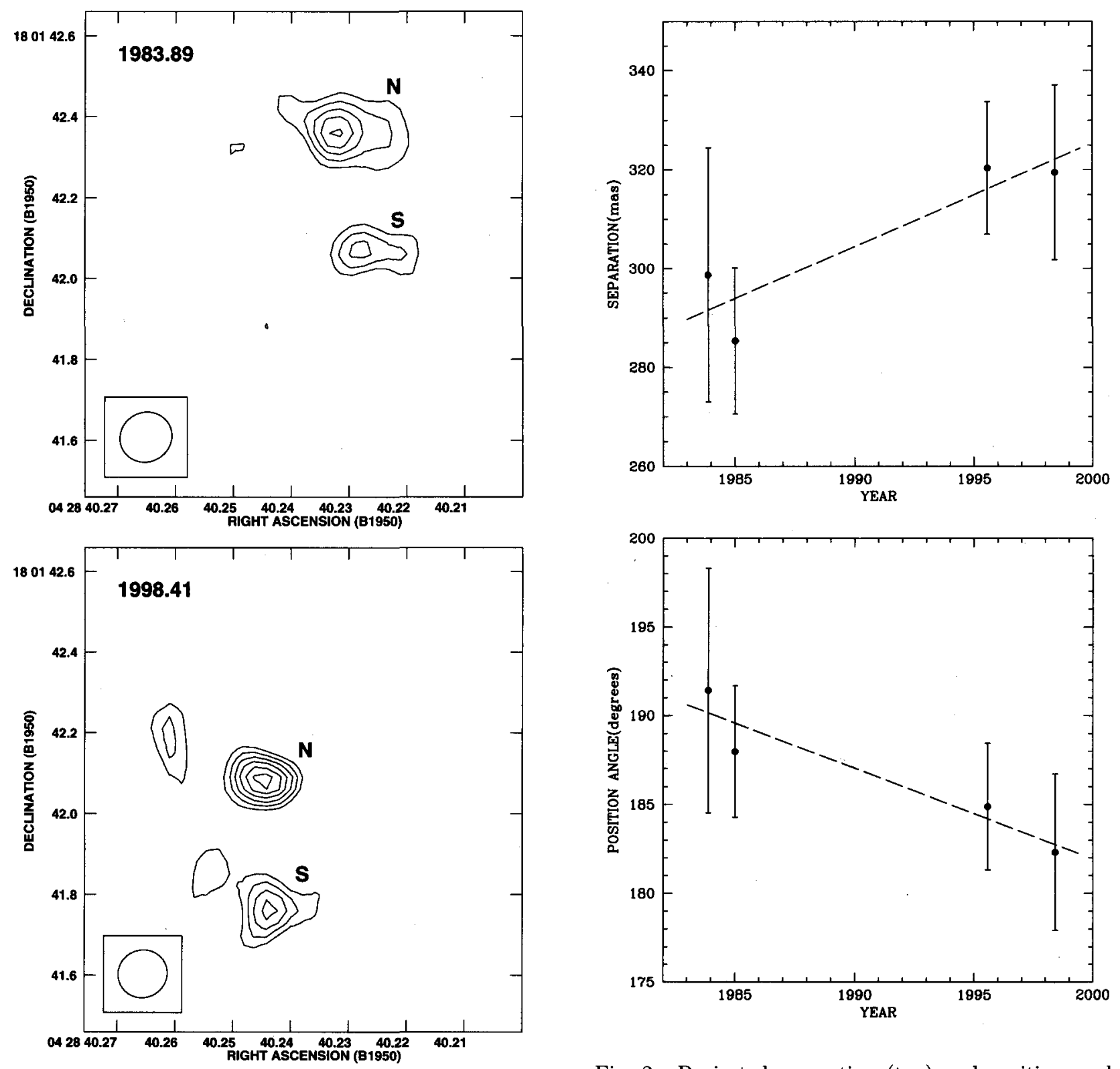

Fig. 1. VLA continuum images of L1551 IRS5 at $2 \mathrm{~cm}$, for the epochs 1983.89 (left) and 1998.41 (right). Contours are $-3,3,4,5,6,7$, and 8 times the rms noise of the images $\left(0.10 \mathrm{mJy} \mathrm{beam}^{-1}\right.$ for 1983.89 and 0.07 for 1998.41). The half-power contours of the beams are shown in the bottom left corner. The north (N) and south (S) components are indicated. Note the large absolute proper motion between the two epochs. The faint components to the east of the $\mathrm{N}$ and $\mathrm{S}$ components in the 1998.41 image are probably due to ejections of ionized gas.

been known to be double since the pioneering observations of Bieging \& Cohen (1985). From $7 \mathrm{~mm}$ VLA observations made at high angular resolution

Fig. 2. Projected separation (top) and position angle (bottom) of the relative orbit of component $\mathrm{S}$ with respect to component $\mathrm{N}$ in L1551 IRS5. The dashed lines are linear least squares fits to the data.

( $\sim 50$ mas), Rodríguez et al. (1998) showed that at this wavelength the emission comes from two compact protoplanetary disks with projected separation of 0.3 ( $\sim 40 \mathrm{AU}$ at a distance of $140 \mathrm{pc}$ ). Rodríguez et al. (1998) also showed that at $2 \mathrm{~cm}$ we are detecting a combination of dust emission from the disks and of free-free emission from ionized ejecta.

Rodríguez et al. (2003) analyzed VLA observations made at $2 \mathrm{~cm}$ in the A configuration at four epochs from 1983 to 1998 . These observations have 

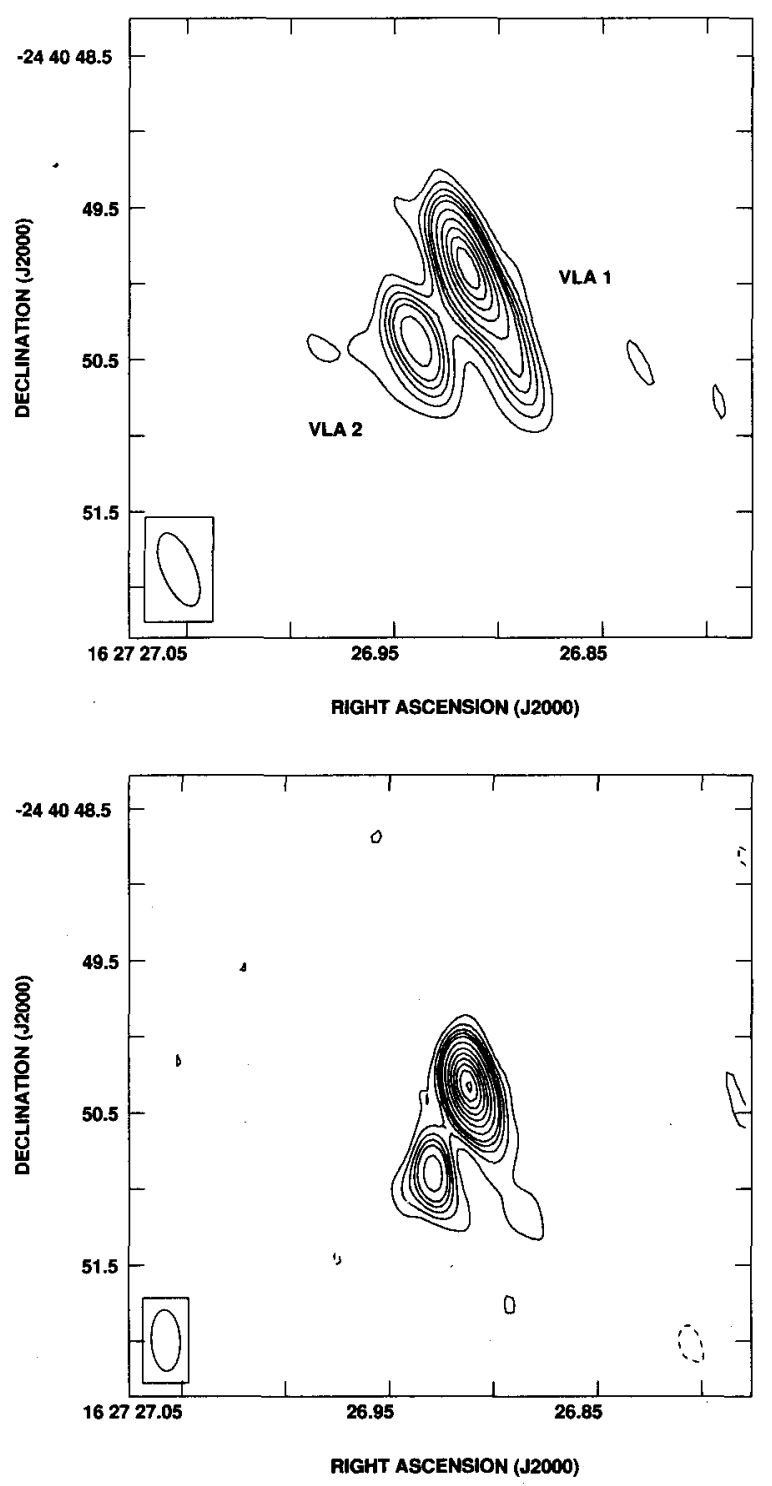

Fig. 3. VLA continuum images of YLW 15 at $.6 \mathrm{~cm}$ for the epochs 1990.41 (top) and 2002.18 (bottom). Contours are $-5,-3,3,5,7,9,11,15, \ldots$, and 45 times the rms noise of the images $\left(18 \mu \mathrm{Jy}\right.$ beam $^{-1}$ for 1990.41 and 22 $\mu \mathrm{Jy}$ beam $^{-1}$ for 2002.18 ). The half-power contour of the beams are shown in the bottom left-hand corner. VLA 1 and VLA 2 are indicated. Note the large absolute proper motions between the two epochs.

an angular resolution of $\sim 0.1$ and resolve the two components of the L1551 IRS5 system, allowing both absolute and relative astrometry to be performed.

In Figure 1 we show the images for the first and last epochs, where the north (N) and south (S) components of L1551 IRS5 have been identified. The absolute proper motion of the sources to the $\mathrm{SE}$ is

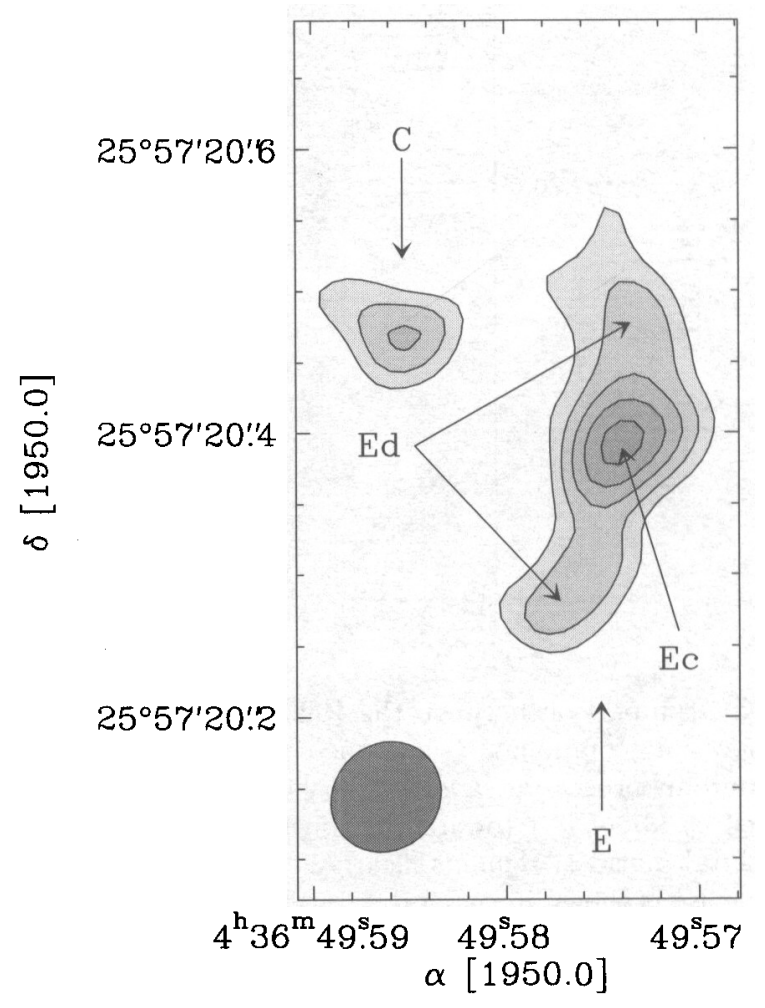

Fig. 4. $7 \mathrm{~mm}$ continuum image of $\mathrm{L} 1527=$ IRAS $04368+2557$. The first contour is at $0.3 \mathrm{mJy}$ beam $^{-1}$, and the contour interval is $0.1 \mathrm{mJy} \mathrm{beam}^{-1}$. The synthesized beam is shown at the bottom left. The components $\mathrm{Ed}, \mathrm{Ec}$ constitute the structure $\mathrm{E}$, that is interpreted to be a disk. The component $\mathrm{C}$ is compact and believed to trace a companion star.

evident. The projected separation and position angle of component $\mathrm{S}$ with respect to component $\mathrm{N}$ are shown in Figure 2. Although the signal-to-noise ratio is modest, a trend is evident in both parameters since the separation shows a monotonic increase with time, while the position angle shows a monotonic decrease. A linear least squares fit to the data gives rates of change for the separation and position angle

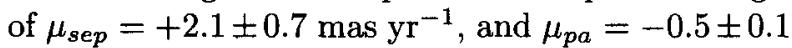
degree $\mathrm{yr}^{-1}$, respectively. The average projected separation for the four epochs is $306 \pm 17$ mas, or $43 \pm 2$ AU.

The observed changes in separation and position angle result in a relative proper motion of $3.4 \pm 0.7$ mas $\mathrm{yr}^{-1}$, which implies a relative velocity in the plane of the sky of $2.3 \pm 0.5 \mathrm{~km} \mathrm{~s}^{-1}$.

A conservative lower limit to the total mass of the system can be derived since $\left(M / M_{\odot}\right) \geq$ $(1 / 2)\left(V_{o b s} / 30 \mathrm{~km} \mathrm{~s}^{-1}\right)^{2}\left(r_{o b s} / A U\right)$, where $V_{o b s}$ and $r_{o b s}$ are the projected relative velocity and sep- 

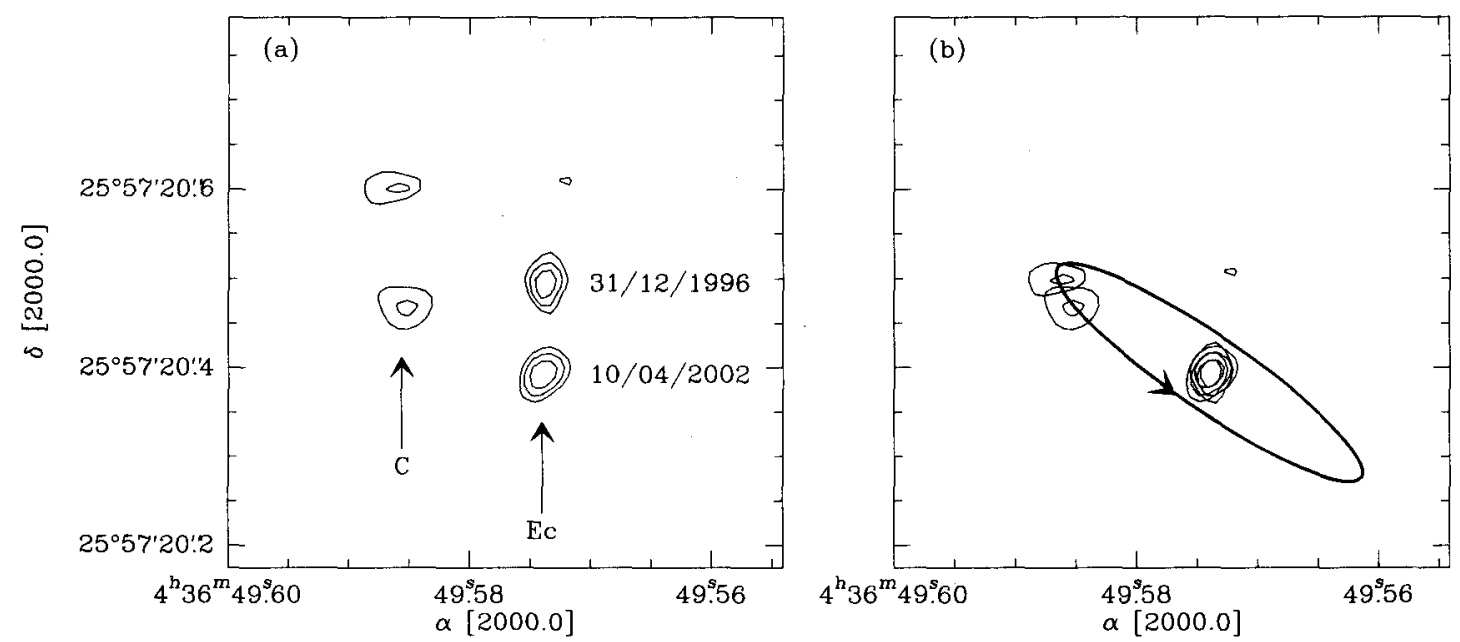

Fig. 5. Comparison between the 1996 and the 2002 data for L1527. For more clarity, the contours have been clipped to show only component $\mathrm{C}$ and the compact component Ec at the center of $\mathrm{E}$. In panel a, where the images are shown without any processing, a large translational shift is evident between the two epochs. In panel b, the 1996 data have been shifted by about 0 . 1 toward the south, to register the images on the position of peak Ec. A residual shift, attributed to orbital motions, remains clearly seen for component C. A possible inclined circular trajectory for the motion of $\mathrm{C}$ around Ec is shown in panel b.

aration. In terms of the observed parameters, the previous equation becomes $\left(M / M_{\odot}\right) \geq$ $1.3\left(\mu / \text { mas } \mathrm{rr}^{-1}\right)^{2}(s / 100 \mathrm{mas})(d / k p c)^{3}$, where $\mu$ is the relative proper motion, $s$ is the angular separation between the members of the binary, and $d$ is the distance to the source. Since $\mu=3.4$ mas $y r^{-1}, s=$ $306 \mathrm{mas}$, and $d=140 p c$, we obtain $M \geq 0.1 M_{\odot}$. We can make a less conservative estimate for the total mass of the system assuming that the plane of the orbit is parallel to the plane of the disks. Furthermore, since the major axes of the disks are approximately in the N-S direction, as is the separation vector, we can conclude that we are observing the binary in an epoch when the velocity in the plane of the sky is at its minimum. We then find that $V_{\text {true }} \simeq\left(V_{\text {obs }} / \cos \left(60^{\circ}\right)\right) \simeq 5 \mathrm{~km} \mathrm{~s}^{-1}$. If we further assume that the orbit is circular, the observed separation will be close to the true separation. We can then estimate the total mass and period of the system to be $M \simeq 1.2 M_{\odot}$ and $P \simeq 260 \mathrm{yr}$, respectively.

\section{2. $Y L W 15$}

YLW 15 (IRS 43) is an infrared protostar with a bolometric luminosity of $10 L_{\odot}$ (Young, Lada, \& Wilking 1986; Wilking, Lada, \& Young 1989) located in L1681B, a small cloud in the Ophiuchus molecular complex. It has been classified as a very young, Class I object by André, Ward-Thompson, \& Barsony (1993) on the basis of its infrared spectral energy distribution and its association with a compact bipolar outflow (Bontemps et al. 1996).

Curiel et al. (2003) reported that the relative astrometry between the two components (separated by $0 " 6$; see Figure 3 ) reveals orbital proper motions that suggest that a lower limit to the total mass and an upper limit to the period of the binary system are $1.7 M_{\odot}$ and $360 \mathrm{yr}$, respectively.

\section{3. $L 1527$}

Using high angular resolution $7 \mathrm{~mm}$ Very Large Array (VLA) observations, Loinard et al. (2002) showed that the low-mass protostellar object IRAS $04368+2557$ in L1527 is composed of two radio sources separated in projection by $25 \mathrm{AU}$ (see Fig. 4). One of the two components is extended in the direction perpendicular to the outflow powered by IRAS $04368+2557$ and is, most likely, a compact accretion disk $(\mathrm{R} \simeq 20 \mathrm{AU})$, similar to those found in L1551 IRS5 by Rodríguez et al. (1998). As in L1551 IRS5, the disk found here is small compared to those around $\mathbf{T}$ Tauri stars. Tidal interactions with nearby companions provide a natural way of truncating disks, and the authors argue that the two millimeter-wavelength sources in L1527 trace a compact binary system, where the disk surrounding one of the components has been truncated by the tidal influence of the other. A comparison between observations obtained in 1996 and 2002 reveals large proper motions, which can be only partly attributed to the overall large-scale motion of the region in 

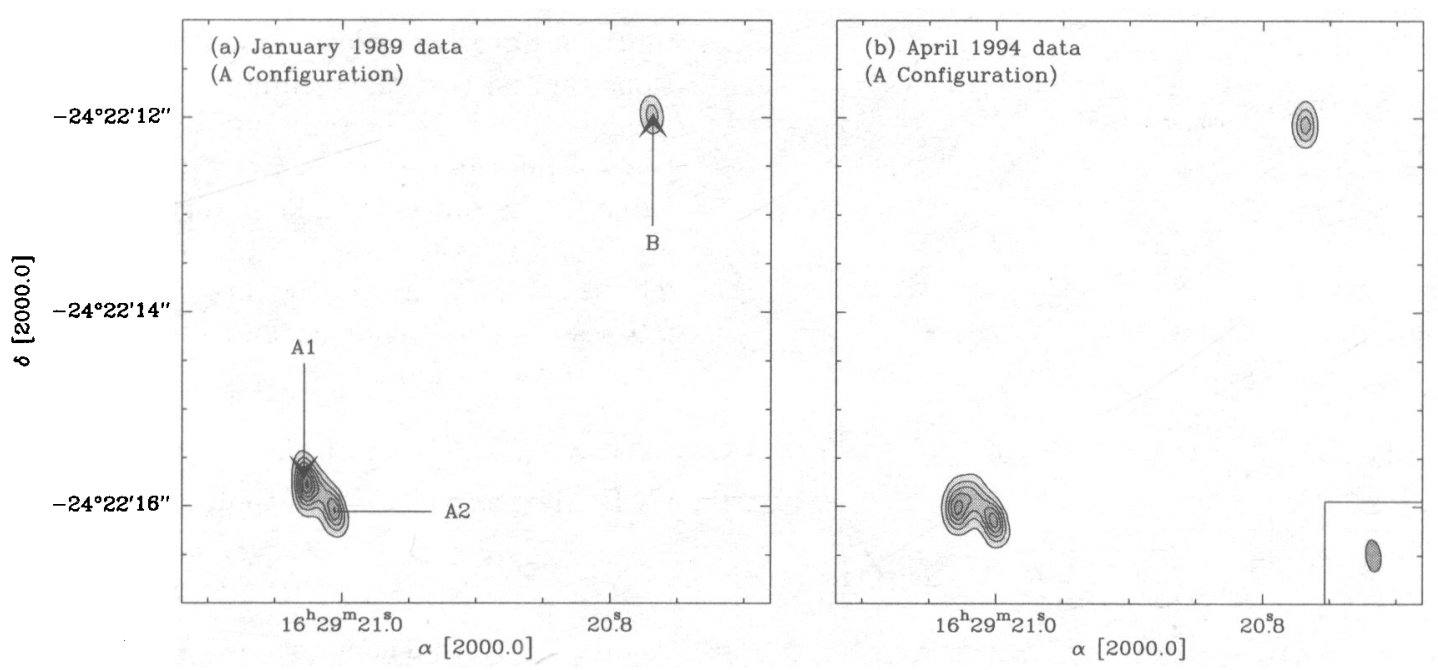

Fig. 6. Comparison of the structure of IRAS 16293-2422 at epochs 1989 and 1994 . (a) 3.6 cm continuum data obtained on January 20, 1989 in A configuration. The first contour and the contour interval are at $0.2 \mathrm{mJy} \mathrm{beam}^{-1}$. The positions of components A1, A2, and B are indicated by the arrows. (b) $3.6 \mathrm{~cm}$ continuum data obtained on April 14, 1994 in A configuration. The first contour and the contour interval are at $0.15 \mathrm{mJy}$ beam $^{-1}$. The synthesized beam (common to both images) is shown at the bottom right of this panel.

which IRAS $04368+2557$ is located. The remaining "residual" proper motions might trace the orbital motion of the binary (see Fig. 5) and would suggest a total mass for the system larger than $0.2 M_{\odot}$ and likely of the order of $0.52 M_{\odot}$.

\section{. 4. TRIPLE SOURCES}

Two of the sources with observed orbital motions appear to be triple: IRAS 16293-2422 and T Tauri.

\subsection{IRAS 16293-2422}

IRAS 16293-2422 (e.g. van Dishoeck et al. 1995, Ceccarelli et al. 2000) is a well-studied low-mass protostellar system located in L1689N (Castets et al. 2001), a small cloud in the $\rho-O$ phiuchus molecular complex, at a distance of $120 \mathrm{pc}$ (Knude \& Hog 1998). High angular resolution radio VLA observations (Wootten 1989) revealed early that it was comprised of two main components (A and $\mathrm{B}$ ) separated by $5^{\prime \prime}(600 \mathrm{AU})$. Component $\mathrm{A}$ is itself comprised of two sub-condensations (A1 and A2) well separated at $2 \mathrm{~cm}$ with the most extended VLA configuration (Wootten 1989). The separation between A1 and A2 is $0 . " 3$ or $35 \mathrm{AU}$.

Loinard (2002) compared high angular resolution VLA $3.6 \mathrm{~cm}$ continuum observations of the protostellar system IRAS 16293-2422 obtained in 1989 and 1994, and showed that the positions of the three VLA sources in IRAS 16293-2422 have changed significantly between the two epochs. The proper motions of two of the components appear to be very similar to one another, and to the proper motions of premain sequence stars in the same direction. Loinard (2002) argued that they correspond to the overall proper motion of the small cloud (L1689N) harboring IRAS 16293-2422. The displacement of the third source (component A1), however, is larger and in a different direction. That component has previously been argued to be a shock between a partly ionized wind and the ambient medium, so some fast motions are not unexpected. It is somewhat puzzling, however, that the direction of the motion does not coincide with the direction of any of the known outflows powered by IRAS $16293-2422$. The relative velocity of about $15 \mathrm{~km} \mathrm{~s}^{-1}$ between components $\mathrm{A} 1$ and $\mathrm{A} 2$ (with a separation of $\sim 30 \mathrm{AU}$ ) implies a total mass for this binary system of about $4 M_{\odot}$, too large for what is known of these systems. At present it is being investigated if $\mathrm{A} 1$ traces a condensation or a real stellar object.

\subsection{T Tauri}

To verify the radio technique, we decided to study an object where optical, near-IR, and radio data were available: $\mathrm{T}$ Tauri. $\mathrm{T}$ Tauri has long been known to be a binary system (Dyck, Simon, \& Zuckerman 1982). The north component, $\mathrm{T}$ Tau $\mathrm{N}$, is considered the prototype for the whole class of low-mass premain sequence stars, and is indeed one of the best studied young stars in the sky. The southern infrared companion ( $\mathrm{T}$ Tau $\mathrm{S}$ ), located about 0.7 arcseconds $(100 \mathrm{AU})$ to the south, is more obscured and not evident in the optical. Recent infrared images of $T$ 


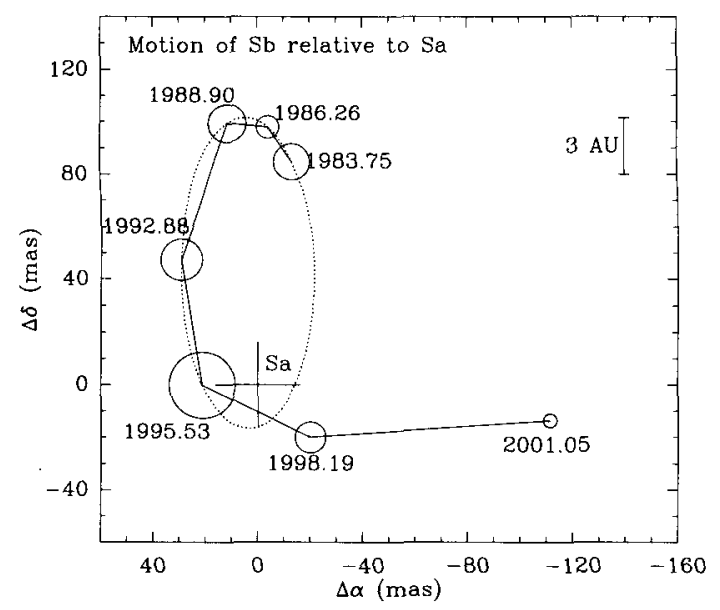

Fig. 7. Orbit of $\mathrm{T}$ Tau $\mathrm{Sb}$ with respect to $\mathrm{T}$ Tau Sa. The numbers give the epoch of the observation and the circles the errors in the relative positions. For this figure we have made weighted averages of the observations made in near epochs $(1983.71+1983.83=1983.75$ and $1988.83+1989.09+1989.10=1988.90)$. To give an idea of the sizes involved, a bar representing $3 \mathrm{AU}$ is shown at the top right. The position of Sa (at $(0,0)$ ) is indicated by a cross, the size of which represents the uncertainty on its position. The dotted ellipse is a fit to the data from the first five epochs (1983.75 to 1995.53).

Tau S obtained by speckle holography showed that, in turn, it is composed of two stars, T Tau Sa and T Tau Sb, separated at the time of the first infrared observations (1997, see Koresko 2000) by a mere 50 mas (7 AU). Subsequent observations (Köhler, Kasper, \& Herbst 2000; Duchêne, Ghez, \& McCabe 2002) showed that the separation between $\mathrm{Sa}$ and $\mathrm{Sb}$ is increasing, and that $\mathrm{Sb}$ is currently moving westward at the fairly large projected velocity of $20 \mathrm{~km} \mathrm{~s}^{-1}$.

Loinard, Rodríguez, \& Rodríguez (2003) reanalyzed archival observations of $\mathrm{T}$ Tauri taken with the Very Large Array during the last 20 years. They concluded that one of the sources $(\mathrm{Sb})$ in the system has moved at moderate speed $\left(5-10 \mathrm{~km} \mathrm{~s}^{-1}\right)$, on an apparently elliptical orbit during the first 15 years of observations, but after a close $(<2$ AU) encounter with the source $\mathrm{Sa}$, it appears to have accelerated westward to about $20 \mathrm{~km} \mathrm{~s}^{-1}$ in the last few years (see Fig. 7). The precise nature of such a dramatic orbital change will be known with about five more years of observations.

\section{CONCLUSIONS}

The few binary of multiple sources whose orbital motions have been determined from radio observations suggest that the technique is important, in particular since it is the only one that can provide this type of information for heavily obscured objects. In three of the objects studied, very reasonable values are found for the masses. However, in IRAS 162932422 and $\mathrm{T}$ Tauri the results suggest either large masses or orbital changes and should be tested with future observations.

I am grateful to DGAPA, UNAM and CONACyT, México for their continuous support.

\section{REFERENCES}

André, P., Ward-Thompson, D., \& Barsony, M. 1993, ApJ, 406, 122

Bieging, J. H. \& Cohen, M. 1985, ApJ, 289, L5

Bontemps, S., André, P., Terebey, S., \& Cabrit, S. 1996, A\&A, 311,858

Castets, A., Ceccarelli, C., Loinard, L., Caux, E., \& Lefloch, B. 2001, A\&A, 375, 40

Ceccarelli, C., Castets, A., Caux, E., Hollenbach, D., Loinard, L., Molinari, S., \& Tielens, A. G. G. M. 2000, $A \& A, 355,1129$

Curiel, S., Girart, J. M., Rodríguez, L. F., \& Cantó, J. 2003, ApJ, 582, L109

Duchêne, G., Ghez, A.M., \& McCabe, C. 2002, ApJ, 568, 771

Dyck, H.M., Simon, T., \& Zuckerman, B. 1982, ApJ, 255, L103

Ghez, A. M., Weinberger, A. J., Neugebauer, G., Matthews, K., \& McCarthy, D. W., Jr. 1995, AJ, 110. 753

Knude, J., \& Hog, E. 1998, A\&A, 338, 897

Köhler, R., Kasper, M., \& Herbst, T. 2000, in Birth and Evolution of Binary Stars, Eds: B. Reipurth and $\mathrm{H}$. Zinnecker (Potsdam: Astrophys. Inst.), 63

Koresko, C. D. 2000, ApJ, 531, L147

Loinard, L. 2002, RevMexAA, 38, 61

Loinard, L., Rodríguez, L. F., D'Alessio, P., Wilner, D. J., \& Ho, P. T. P. 2002, ApJ, 581, L109

Loinard, L., Rodríguez, L. F., \& Rodríguez, M. I. 2003, ApJ, 587, L47

Rodríguez, L. F. et al. 1998, Nature, 395, 355

Rodríguez, L. F., Curiel, S., Cantó, J., Loinard, L., Raga, A. C., \& Torrelles, J. M. 2003, ApJ, 583, 330

van Dishoeck, E.F., Blake, G.A., Jansen, D.J., \& Groesbeck, T.D. 1995, ApJ, 447, 760

Wilking, B. A., Lada, C. J., \& Young, E. T. 1989, ApJ, 340,823

Wootten, H.A. 1989, ApJ, 337, 858

Young, E. T., Lada, C. J., \& Wilking, B. A. 1986, ApJ, 304, L45

Luis F. Rodríguez: Centro de Radioastronomía y Astrofísica, Universidad Nacional Autónoma de México, Apartado Postal 3-72, 58090 Morelia, Michoacán, México (l.rodriguez@astrosmo.unam.mx). 


\section{DISCUSSION}

Hummel - There is a paper in press by G. Johnston et al. on T Tau based on the same VLA data, and they came to a different conclusion regarding the orbit.

Mathieu - What is the projected separation between T Tau SA and SB at closest approach? If this is an ejection, what would this imply for the properties of the close binary? Might the prediction that it is a close binary be tested with near-infrared spectroscopy?

Rodríguez - The projected separation is about $2 \mathrm{AU}$. For the ejection to be feasible, the separation of the close binary should be about $1 \mathrm{AU}$ and the period of the order of a year. The presumed close binary is now being observed with precise near-infrared spectroscopy to test if it is a binary.

Zinnecker - You argued that for the stellar masses involved the observed bolometric luminosity $\left(30 L_{\odot}\right)$ of L1551-IRS5 is too high to be due to stellar radiation and must be due to accretion. However, you referred only to the main sequence and not to the pre-main sequence luminosity. Can you rule out a significant pre-MS contribution?

Rodríguez - These extremely young stars are probably deriving their luminosity from a combination of thermonuclear reactions, contraction, and accretion. However, given their large luminosities, we believe that accretion is the main contributor.

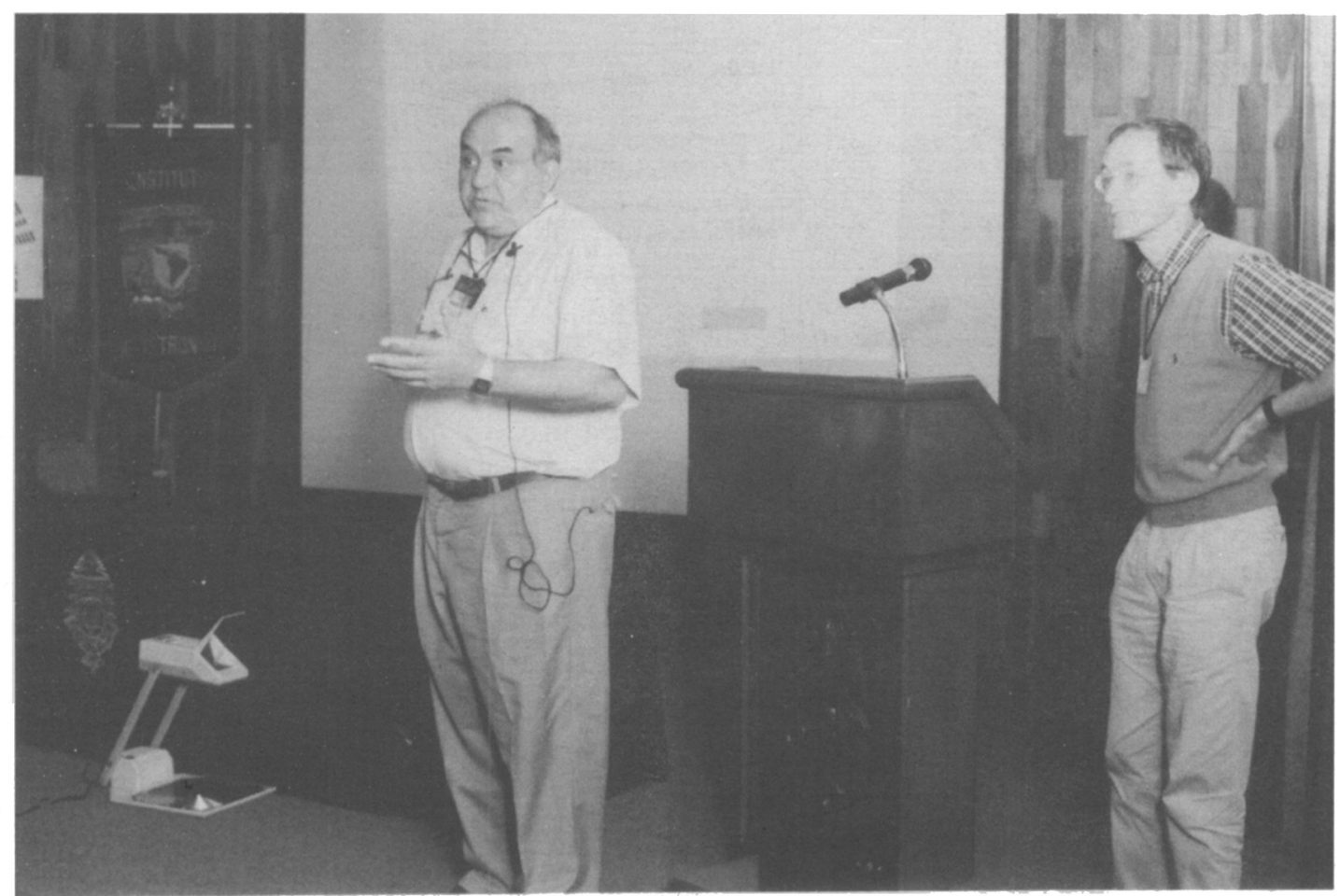

Luis F. Rodríguez and A. Tokovinin. 